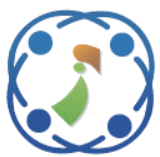

\title{
An Adaptive Sub Band Filtering Based Medical Image Fusion Through Non- subsampled Contourlet Transform
}

\author{
Devanna Harijana $^{1^{*}} \quad$ Arthur Edwin Satish Kumar Gandluru $^{2} \quad$ Giri Prasad Mahendra Nanjappa ${ }^{3}$ \\ ${ }^{1}$ Department of Electronics and Communication Engineering, \\ Jawaharlal Nehru Technological University Anantapur, Ananthapuramu, A.P, India \\ ${ }^{2}$ Department of Electronics and Communication Engineering, \\ Vardhaman College of Engineering, Hyderabad, Telangana, India \\ ${ }^{3}$ Department of Electronics and Communication Engineering, \\ Jawaharlal Nehru Technological University College of Engineering, Anantapuramu, A.P, India \\ * Corresponding author's Email: nayakantidev@gmail.com
}

\begin{abstract}
Medical image fusion is a powerful tool in the medical oriented applications such as diagnosis, treatment planning and image-guided radiotherapy. In this paper, a new medical image fusion approach is proposed based on Non-Subsampled Contourlet Transform (NSCT) and Adaptive sub band filtering. Though there are so many approaches proposed in earlier to find a more and clear fused image, they didn't focus on the computational complexity. Since the computational complexity directly related to the feature count, the rise in the level number of sub band images rises the computational complexity. The adaptive sub band filtering mechanism retrieves the informative sub band images only form all the obtained high frequency sub band images. Further to improve the fusion performance, the proposed approach fuses the low frequency subband images based on phase congruency rule and the high frequency subband images based on Log-Gabor energy rule. Visually and quantitatively experimental results indicate that the proposed fusion method can obtain more effective and accurate fusion results of medical images than other algorithms.
\end{abstract}

Keywords: Medical image fusion, NSCT, Sub band filtering, Phase congruency, Log-Gabor energy, Mutual information, $Q^{(A B / F)}$.

\section{Introduction}

Medical imaging attracts more and more attention due to the increasing requirements of clinic investigation and disease diagnosis. Owing to different imaging mechanisms, medical images of different modals provide a variety of complementary information about the human body in a limited domain. For example, the computed tomography (CT) images provide better information on dense tissue, the positron emission tomography (PET) images supply better information on blood flow and tumor activity with low space resolution, and the magnetic resonance (MR) images show better information on soft tissue. Moreover, the MR-T1 images give more detailed information about anatomical structures; whereas the MR-T2 images contain a greater contrast between the normal and abnormal tissues. However, single multiple modality cannot satisfy the demand of images with high resolution and visualization for disease diagnosis. In this regard, medical image fusion [1-4] is a useful and powerful technique for integrating complementary information from multimodality images to improve the diagnostic accuracy. Besides, the fused images are more suitable for assisting the doctors in diagnosis and treatment planning: fusing MR and CT images can generate the images which can describe the soft tissue and bone in order to concurrently represent anatomical and physiological features of the human body. MR-T1 and MR-T2 images are fused to segment white matter lesions 
and guide neurosurgical resection of epileptogenic lesions. In oncology, the combined PET/CT imaging is helpful to view the anatomical, physiological characteristics and the tumor activity. More than that, medical image fusion not only helps in diagnosing diseases but also reduces the storage cost.

Image fusion methods are usually divided into transform domain [5-7] and spatial domain [8-10] techniques. Fusion methods in the spatial domain are directly on pixel graylevel or color space from the source images for fusion operation, so the spatial domain fusion methods are also known as singlescale fusion method. For transform domain based methods, each source image is first decomposed into a sequence of images through a particular mathematical transformation.Then; the fused coefficients are obtained through some fusion rules for combination. Finally, the fusion image is obtained by means of a mathematical inverse transform. Thus, the transform domain fusion methods are also known as Multi-scale fusion methods.There is evidence that Multi Scale Transform (MST) with signal decomposition is similar to the human visual system (HVS). Compared with spatial-based methods, the methods using MST successfully overcome the disadvantages. The reason for this is that the decomposition coefficients of MST.consider the detail of the input images and selects them out to compose fused image.

The recent studies over medical image fusion declared that the Non-Subsampled Contourlet Transform obtained an outstanding performance, both quantitatively and qualitatively. Though the Non-Subsampled Contourlet Transform based medical image fusion achieved an effective fusion results, the computational complexity observed to be more due to the architectural configuration. The earlier approaches considered the entire feature set of all the sub band images for the fusion, for both low frequencies and high frequencies. This makes the fused image more informative but the complexity will increase substantially. If the entire feature set is not considered for fusion, the fused image results is poor informative by which the medical image analysis will goes wrong. In addition, most of the earlier image fusion approaches are based on an assumption that the source images are noise free. However in practice the source images are often corrupted by noises during the image capturing. Though there is an approach proposed in [21] for noise cleaning the method is observed to be complicated and the computational time take is more.

To overcome these limitations, this paper proposes a new medical image fusion approach based on the Non-Subsampled Contourlet Transform and sub band adaptive filtering which makes the fused image more informative with less computational complexity. After decomposing the source image through Non-Subsampled Contourlet Transform, the proposed approach applies the an adaptive sub band adaptive filtering mechanism to extract only the informative features form the high frequency sub band images. Further the obtained features are fused through low frequency fusion rule and high frequency fusion rule to obtain final fused image.

Rest of the paper is organized as follows: The related literature survey of the proposed work is illustrated in section II. The basic architecture configuration of Non-Subsampled Contourlet Transform is represented in section III. The complete details of proposed approach are illustrated in section IV. Section V illustrates the details of performance evaluation and finally the section VI concludes the paper.

\section{Literature survey}

Based on the methodology of fusion process, the earlier approaches are categorized into two categories as pixel based image fusion and transform based image fusion. The simplest spatialbased method is to take the average of the input images pixel by pixel. However, along with its simplicity, this method leads to several undesirable side effects, such as reduced contrast. To improve the quality of fused image, various approaches are propose in earlier based on the block division if source images. Here the source images are initially decomposed into blocks and the optimal blocks are chosen for the fusion. The motivation of this methodology lies in the fact that an optimized block size could be more effective than a fixed block size. This type of algorithm may not only improve the convergence between each pixel in the fused image but may also easily produce block effect. And also the finding of a suitable block-size is a problem. A large block is more likely to contain portions from both focused and defocused regions. This may lead to selection of considerable amount of defocused regions. On the other hand, small blocks do not vary much in relative contrast and hence difficult to choose from. Moreover, small blocks are more affected by misregistration problems. To solve these issues, a novel optimal method for multi-focus image fusion using differential evolution algorithm is presented in [10]. The source images are first decomposed into blocks. Then, the sharper blocks are selected by employing a sharpness criterion 
function. The selected blocks are finally combined to construct the fused image. Similarly the quad-tree structure method is proposed in [11] to solve the problem of how to determine the size of sub-blocks. Further two more block based approaches are proposed in [12] and [13] to evaluate the local content (sharp) information of the input source images by which the blocking effect in the fused image will reduces efficiently. Though these approaches achieved an efficient fusion performance, the blocking effect is not eliminated completely. Another region segmentation approach is proposed in [14] to find the regions through the morphological filtering. Then, image matting technique is applied to obtain the accurate focused region of each source image. Finally, the focused regions are combined together to construct the fused image. Through image matting, the proposed fusion algorithm combines the focus information and the correlations between nearby pixels together [15].However, these methods may generate artificial information and discontinuous phenomena at the boundaries of focused regions because the boundary cannot be determined accurately. These effects will reduce the visual fidelity of the fused image.

To achieve more efficient results, the medical image fusion is shifted towards the transform domain through the accomplishment of MST, including the discrete wavelet transform (DWT) [3, 7, 37], framelet transform [16], contourlet transform [17], and non-sub sampled contourlet transform (NSCT) $[1,4,6]$. By focusing on the properties of wavelet filters, some extended wavelet based image fusion approaches are proposed based on Wavelet Packet Transform (WPT) [18] and Wavelet Frame Transform (WFT) [19]. Wavelet transform suffers from lack of shift invariance \& poor directionality and Stationary Wavelet Transform and Wavelet Packet Transform overcome these disadvantages. Further the wavelet frame transform is aliasing free and translation invariant. In [37], a new image fusion approach is proposed based on the Discrete Wavelet transform and type-2 fuzzy logic. Here the main is avoiding the extra noise in the fused image. In this method, source images are decomposed into low-level subband, high-level subbands using DWT. Next, low-level sub-images are fused using type-2 fuzzy fusion rule and high-level sub-images are fused using average fusion rule. Finally, inverse DWT is applied on the fused components to obtain the fused image. However, wavelet transform cannot effectively represent the line singularities and plane singularities of the images.

To overcome these shortcomings with wavelet transform, the further research is focused through the contourlet transform and Non-Subsampled Contourlet Transform. The main difference between the Contourlet Transform and Non-Subsampled Contourlet Transform is shift invariant property. Recently Hui Huang et al., [20] propose a novel image fusion algorithm that combines nonlinear approximation of contourlet transform [26] with image regional features. The most important coefficient bands of the contourlet sparse matrix are retained by nonlinear approximation.However, the up- and down-sampling process of Contourlet decomposition and reconstruction results in the CT lacking shift-invariance and having pseudo-Gibbs phenomena in the fused image. The NonSubsampled Contourlet Transform inherits the advantages of Contourlet Transform, while also possessing shift-invariance and effectively suppressing Pseudo-Gibbs phenomena. Hence NonSubsampled Contourlet Transform is chosen as a prominent transform for multimodal medical image fusion and so many approaches are proposed based on Non-Subsampled Contourlet Transform [22-25, 27].

\section{Non-subsampled contourlet transform}

The Non-Subsampled Contourlet Transform [28] is developed based on the theory of contourlet transform only. Non-Subsampled Contourlet Transform is advantageous in the provision of shift invariance, boosts the directional selectivity and reduces the significance of pseudo-Gibbs phenomena effectively. The decomposition process of the Non-Subsampled Contourlet Transform is divided into two phases, i.e., the Non-sub Sampled Pyramids (NSP) and the Non-sub Sampled Directional Filter Bank (NSDFB). The former performs multiscale decomposition and the later provide direction decomposition. The NSP divides image into a low frequency sub band and a high frequency sub band in each level. For a given $k$ level of decomposition the NSP generates $\mathrm{k}+1$ sub-band images, consists of one low frequency sub band image and the remaining $\mathrm{k}$ sub band images are high frequency sub band images. Subsequently, the NSDFB divides the high frequency sub band image into directional sub band images. For a given level of decomposition 1, 21 directional sub band images will be obtained for a particular high frequency sub band image.

After the low frequency component is decomposed iteratively by the same way, an image is finally decomposed into one low frequency sub image and a series of high frequency directional sub 


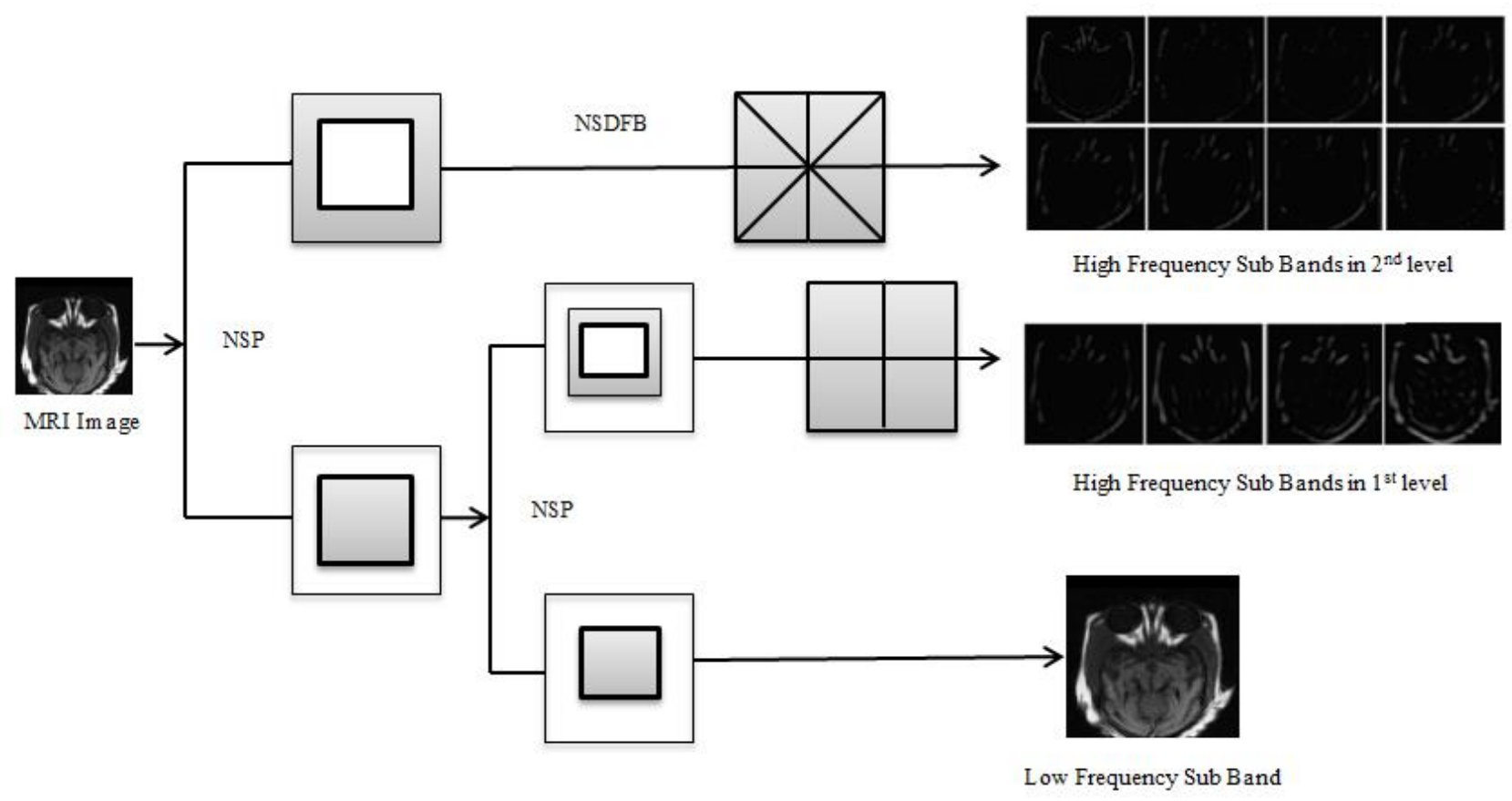

Figure.1 MRI Image decomposition through Non-Subsampled Contourlet Transform for two levels

band images $\left(\sum_{j=1}^{k} 2^{l_{j}}\right)$, where in $l_{j}$ denotes the number of decomposition directions at the $j$ scale. Figure.1 represents the schematic of Non-Subsampled Contourlet Transform. Hence the NSDFB offers more accurate directional information through the bands obtained in multi-directional orientations to produce more accurate results. Thus, the Non-Subsampled Contourlet Transform ensures the optimal frequency selectivity and also an essential shift invariance property on the aspect of non-subsampled operation. Here an important to note that is the dimensions of obtained sub-images is in identical fashion. In addition, the Non-Subsampled Contourlet Transform also reduces the misregistration effects over the obtained results. Thus, the proposed model considered Non-Subsampled Contourlet Transform for medical image effusion.

\section{Adaptive sub band filtering (ASF)}

In various signal and image processing applications, refinement of a signal/image is made to achieve higher level of accuracy. In the process of band decomposition, it is observed that, finer details reveal more clear information's than the original processing signal. However as the band decomposition increases, the probability of redundancy among different bands increases. This redundancy of information increases the processing overhead, and intern makes the system slower. Hence it is required to have an adaptive band selection process for extracting the actual informative band from the processed bands. In the process of signal processing an adaptive band selection process for subband coding was made in [29]. However no such approach of band selection is observed in image coding. With reference to band selection process in this work the process of adaptive band selection is developed for NonSubsampled Contourlet Transform sub band images. For this purpose, initially the source images are decomposed through Non-Subsampled Contourlet Transform and then the adaptive sub band filtering is accomplished over the obtained high frequency sub bands to obtain only informative bands. The process of adaptive sub band filtering is illustrated in the following section.

The ASF operation is based on the LMS-type adaptive filter. The converged of such filter is based on the optimization of this LMS function, wherein weight functions are used to optimize the mean error. To converge the cost function faster in [30] a Normalized ASF (NASF) is proposed. In this approach the convergence speed is increased by increasing the number of subband filters while maintaining the same level of steady-state error. However, it suffers from huge complexity when used in adapting an extremely long unknown system. To overcome this problem in [31] a dynamic selection based NASF (DS-NASF) scheme is proposed. This approach sorts out a subset of the subband filters contributing to convergence performance and utilizes those in updating the adaptive filter weight. This approach dynamically selects the subband filters so as to fulfill the largest decrease of the successive mean square deviations 
(MSDs) at every iteration. This approach reduces the computational complexity of the conventional ASF with critical sampling while maintaining its selection performance. The operational approach for the conventional DS-ASF approach [30] is as outlined.

In a ASF system the desired band $d(n)$ that originates from an its lowering band is defined by,

$$
d(n)=u(n) W^{o}+v(n)
$$

Where $W^{o}$ is an unknown column vector to be identified with an adaptive filter, $v(i)$ corresponds to a variance $\sigma_{v}{ }^{2}$ for each band, and $u(n)$ denotes a row input vector with length $\mathrm{M}$ defined as;

$$
u(n)=[u(n) u(n-1) \ldots u(n-M+1)]
$$

In the process of adaptive selection, the Normalized ASF (NASF) [31] approach was proposed.In this approach the source image sample is partitioned into $N$ subbands by the Non-subsampled directional filter banks (NSDFB). The resulting subband signals are then critically decimated to a lower sampling rate relative to their demanded bandwidth. The original signal $d(n)$ is decimated to $k$ signals and the decimated filter output at each subband is defined as;

$$
y_{i, D}(k)=u_{i}(k) w(k)
$$

Where, $u_{i}(k)$ is a $1 \times M$ row such that, $u_{i}(k)=$ $\left[u_{i}(k N), u_{i}(k N-1), \ldots \ldots, u_{i}(k N-M+1)\right]$ and $w(k)=\left[w_{0}(k), w_{1}(k), \ldots \ldots, w_{M-1}(k)\right]^{T}$ denotes the estimated weight value and the decimated band error is then defined by,

$$
\begin{array}{r}
e_{i, D}(k)=d_{i, D}(k)-y_{i, D}(k)=d_{i, D}(k)- \\
u_{i}(k) w(k)
\end{array}
$$

Where $\quad d_{i, D}(k)=d_{i}(k N)$ is the reference information at each band. In the process of NASF the weight optimization is defined as,

$$
w(k+1)=w(k)+\mu \sum_{i=0}^{N-1} \frac{u_{i}^{T}(k)}{\left\|u_{i}(k)\right\|^{2}} e_{i, D}(k)
$$

Where $\mu$ is the step size. This weight is used to optimize the band selection process where in it takes a large computation to converge for the optimization. To overcome this issue in [30] a MSD based weight optimization is proposed. In this DS-NASF approach the largest decrease of the MSDs between successive iterations is used.
Hence the weight error vector is then defined as, $\widetilde{w}(k)=w^{o}-w(k)$. The weight optimization is then defined as,

$$
\widetilde{w}(k+1)=\widetilde{w}(k)-\mu \sum_{i=0}^{N-1} \frac{u_{i}^{T}(k)}{\left\|u_{i}(k)\right\|^{2}} e_{i, D}(k)
$$

Using this weight vector and taking the expectation a MSD is computed which satisfies the absolute expectation as,

$$
\begin{aligned}
& E\|\widetilde{w}(k+1)\|^{2} \\
& =E\|\widetilde{w}(k)\|^{2}+\mu^{2} E\left[\sum_{i=0}^{N-1} \frac{e_{i, d}^{2}(k)}{\left\|u_{i}(k)\right\|^{2}}\right] \\
& -\quad 2 \mu E\left[\sum_{i=0}^{N-1} \frac{u_{i}(k) \widetilde{w}(k) e_{i, D}(k)}{\left\|u_{i}(k)\right\|^{2}}\right] \\
& \triangleq E\|\widetilde{w}(k)\|^{2}
\end{aligned}
$$

Where

$$
\Delta=\mu \sum_{i=0}^{N-1}\left(2 E\left[\frac{u_{i}(k) \widetilde{w}(k) e_{i, D}(k)}{\left\|u_{i}(k)\right\|^{2}}\right]-\mu E\left[\frac{e_{i, d}^{2}(k)}{\left\|u_{i}(k)\right\|^{2}}\right]\right)
$$

Defines the difference of MSDs between two successive bands. With bands having minimum MSD is then chosen to have a selective band for processing rather to all decomposed bands. This band selection process reduces the processing coefficient with minimum deviation due to the selecting criterion of minimum MSD value.

Further the obtained informative high frequencies only are processed for fusion based on the maximum absolute rule. The further fusion process in illustrated as follows.

\section{Fusion framework}

Due to the beneficial properties of NonSubsampled Contourlet Transform, it is chosen for decomposition of source images and then the redundancy between high frequency bands is reduced through the adaptive sub band filtering. For every source image, the Non-Subsampled Contourlet Transform decomposes it into one low frequency band and $N$ number of high frequency bands. Further the bands are processed for fusion through fusion rule. Here the fusion rules also play an important role in the medical image fusion. Hence the choice of fusion rules is also an important that depends on the sub bands obtained. In a MSTbased image fusion algorithm, such as the NonSubsampled Contourlet Transform domain, one of the most important things for improving fusion 
quality is to design the fusion rules, which will affect the performance of the fusion algorithm remarkably. In order to achieve better performance, Phase congruency [4] and local Log-Gabor energy [32] are and used to merge the low- and highfrequency coefficients, respectively. The step by step process of proposed image fusion frame work is represented below:

Consider two medical images A and B captured under two different models. The proposed image fusion framework is illustrated step by step as follows;

Step 1:Decompose the two source images A and B through Non-Subsampled Contourlet Transform to attain one low frequency band and $t$ high frequency bands for a direction $l$. Let $L F_{k, l}^{A}$ and $L F_{k, l}^{B}$ are the obtained low frequency bands of source image A and source image B respectively. Similarly, $\left\{H F_{k, l}^{A}\right\}$ and $\left\{H F_{k, l}^{B}\right\}$ are the obtained set of high frequency bands of source image A and source image B respectively.

Step 2: Fuse the low frequency coefficients of both image $\mathrm{A}$ and $\mathrm{B}$ through the proposed fusion rule to obtain the composite low frequency sub image of a fused image.

Generally, the low frequency information of source images is fused according to the most popular averaging rule. Though it is easier for fusion, the quality of fused images should not be that much better and it does not represents the entire information of both source images. However, this rule reduced contrast in the fused images and cannot give the fused sub image of high quality for medical image. Therefore, the criterion based on the phase congruency [4] that is employed to fuse the lowfrequency coefficients. The fusion rule for the lowfrequency sub bands is defined as

$$
\begin{aligned}
& L F^{F}(x, y)= \\
& \begin{cases}L F^{A}(x, y) & \text { if } A_{c}^{A \theta}(x, y)>A_{c}^{B \theta}(x, y) \\
L F^{B}(x, y) & \text { if } A_{c}^{A \theta}(x, y)<A_{c}^{B \theta}(x, y) \\
\frac{1}{2}\left(L F^{A}(x, y)+L F^{B}(x, y)\right) & \text { if } A_{c}^{A \theta}(x, y)=A_{c}^{B \theta}(x, y)\end{cases}
\end{aligned}
$$

Where $A_{c}^{A \theta}(x, y), A_{c}^{B \theta}(x, y)$ phase congruency is extracted from low-frequency sub images of the source images $A$ and Brespectively.

High frequency sub bands correspond to detailed information in these regions such as edges, lines, and corners. Because of different imaging modalities contain redundant and complementary information of each other; the purpose of selection rule is mainly to capture salient information of the source images as much as possible. Larger absolute selection rule is not suitable for medical image fusion, because it works well on this premise that only an original image provides good pixel at each corresponding location; thus vast complementary information will be lost when it is used for MIF. One more issue, the noise coefficients are also resembles the nature of high frequency coefficients. Thus, there is a chance of miscalculation of noise as high frequency coefficients. Therefore, the criterion based on Log-Gabor energy [32] is accomplished to fuse high-frequency coefficients. The fusion rule for the high-frequency sub bands is defined as

$$
\begin{aligned}
& H F^{F}(x, y)= \\
& \begin{cases}H F^{A}(x, y) & \text { if } E^{A}(x, y) \geq E^{B}(x, y) \\
H F^{B}(x, y) & \text { if } E^{A}(x, y)<E^{B}(x, y)\end{cases}
\end{aligned}
$$

Where $E^{A}(x, y)$ and $E^{B}(x, y)$ are the Log-Gabor energies of the high frequency sub images extracted from source image A and source image B respectively through Non-Subsampled Contourlet Transform.

Step 3: Reconstruct the fused image through inverse Non-Subsampled Contourlet Transform form the obtained low frequency and high frequency sub images.

\section{Simulation results}

This section illustrates the details of performance evaluation of proposed framework quantitatively and qualitatively. To verify the performance of proposed approach, an extensive simulation is carried out over various types of medical images and like MRI, CT, MR-T1 and MRT2. Here the source images of size $256 * 256$ are considered and the simulation is carried through MATLAB software. The obtained fused images are shown in Figs. 3-5.

In this paper, five objective evaluation measurements parameters are adopted to evaluate the fusion performance. There are local quality index $\left(Q_{0}\right)$ [34],weighted fusion quality index $\left(Q_{W}\right)$ [34], edge-dependent fusion quality index $\left(Q_{E}\right)$ [34], $Q_{A B / F}[35]$ which measures the transmission of edge and visual information from source images to fused images, and mutual information (MI) [36]which computes the information transformed from the source images to the fused images. The range of $Q_{0}$, $Q_{W}, Q_{E}, Q_{A B / F}$ lies between 0 and 1 and the range of MI is above 1 and it varies from image to image. 

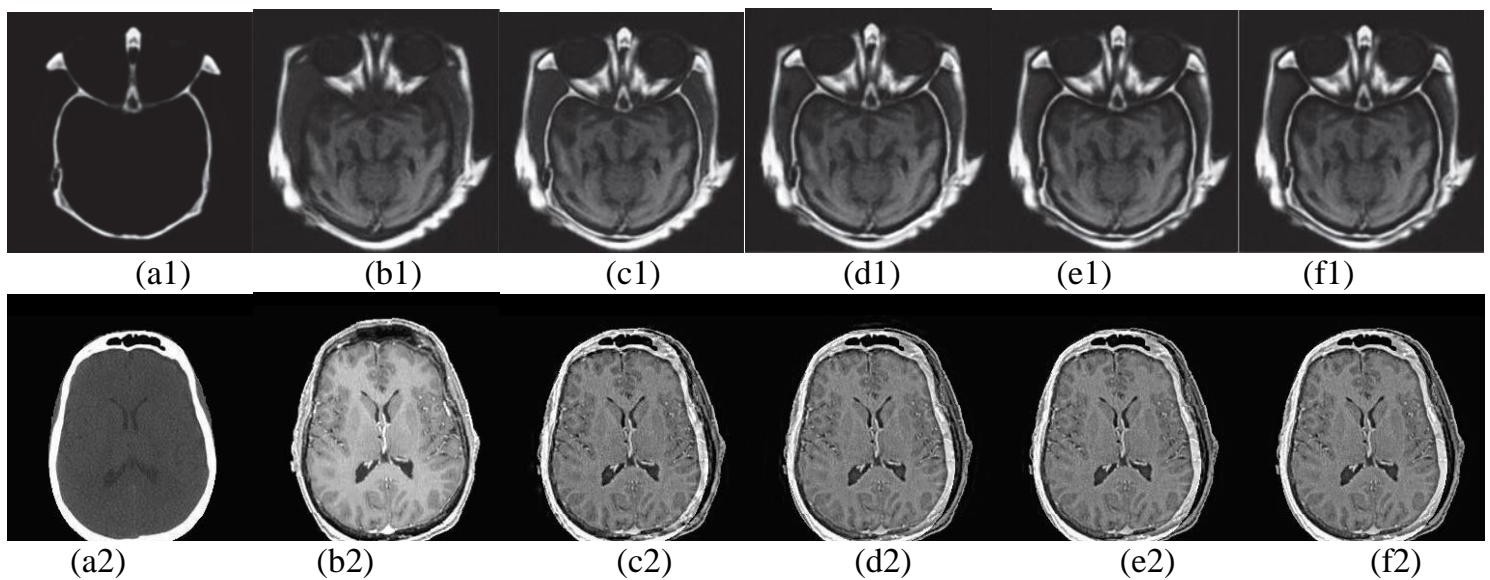

Figure.2. Obtained results: (a1)-(a2) CT images, (a3)-(a4) MR-T1 images, (b1)-(b2) MRI images, (b3)-(b4) MR-T2 images, (c1)-(c4) fused by non-subsampled contourlet transform-1, (d1)-(d4) fused by non-subsampled contourlet transform-2, (e1)-(e4) fused through non-subsampled contourlet transform -3, and (f1)-(f4) fused through proposed approach
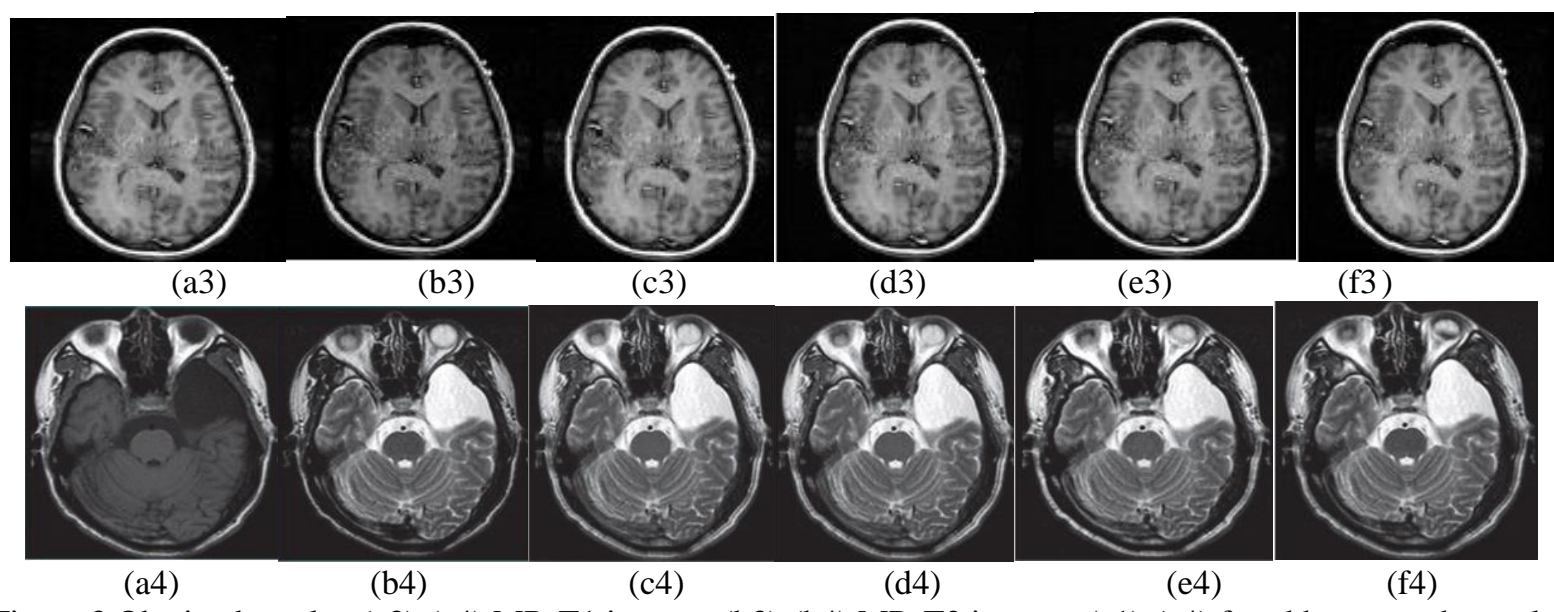

Figure.3 Obtained results: (a3)-(a4) MR-T1 images, (b3)-(b4) MR-T2 images, (c1)-(c4) fused by non-subsampled contourlet transform -1, (d1)-(d4) fused by non-subsampled contourlet transform -2, (e1)-(e4) fused through nonsubsampled contourlet transform -3 , and (f1)-(f4) fused through proposed approach

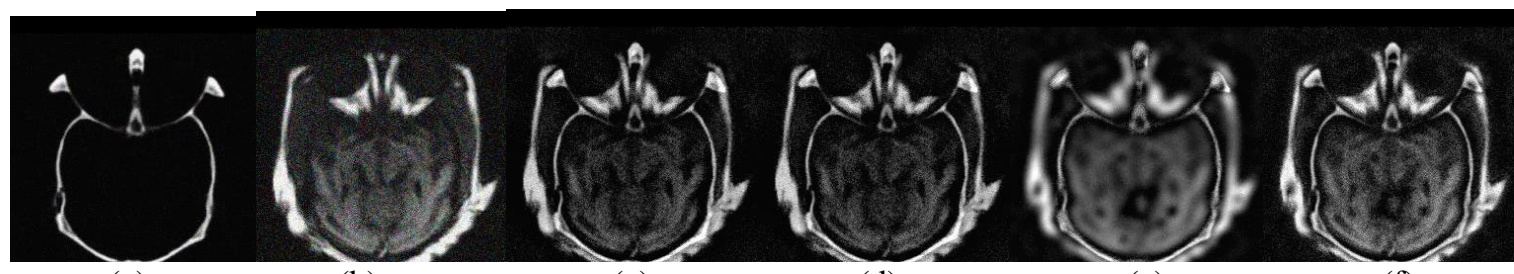

(a)

(b)

(c)

(d)

(e)

(f)

Figure.4 Fused results in the case of external noise: (a) CT image with gaussian noise 0.001 , (b) MRI image with noise variance 0.001 , (c) fused through non-subsampled contourlet transform -1, (d) fused through non-subsampled contourlet transform -2, (e) fused through non-subsampled contourlet transform -3, and (f) fused through proposed approach

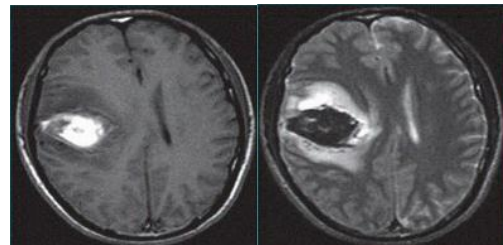

(a)

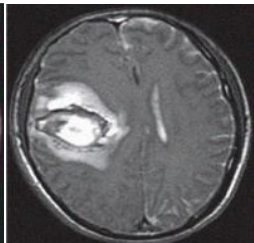

(c)

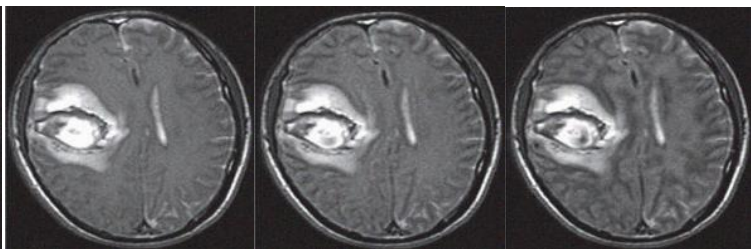

(d) (e)

Figure.5 Fused results in the case of external noise: (a) MR-T1 image with gaussian noise 0.001, (b) MR-T2 image with noise variance 0.001, (c) fused through Non-Subsampled Contourlet Transform -1, (d) fused through non-subsampled contourlet transform -2, (e) fused through non-subsampled contourlet transform -3 , and (f) fused through proposed approach 
Table 1. The objective evaluation results for CT and MRI image dataset

\begin{tabular}{|c|c|c|c|c|c|}
\hline Image Set & Metric & NSCT-1 [1] & NSCT-2 [4] & NSCT-3 [6] & Proposed \\
\hline \multirow{4}{*}{ Image set 1 } & $Q_{0}$ & 0.5960 & 0.5972 & 0.5998 & 0.6471 \\
\cline { 2 - 6 } & $Q_{W}$ & 0.7514 & 0.7536 & 0.7544 & 0.7596 \\
\cline { 2 - 6 } & $Q_{E}$ & 0.5703 & 0.5729 & 0.5768 & 0.5844 \\
\cline { 2 - 6 } & $Q_{A B / F}$ & 0.5812 & 0.5843 & 0.5875 & 0.5963 \\
\cline { 2 - 6 } & $\mathrm{MI}$ & 4.6613 & 4.6696 & 4.6709 & 4.6759 \\
\cline { 2 - 6 } & $\mathrm{CT}(\mathrm{sec})$ & 2.3654 & 3.1274 & 3.2471 & 2.0027 \\
\hline \multirow{4}{*}{ Image set 2 } & $Q_{0}$ & 0.5973 & 0.5985 & 0.6011 & 0.6484 \\
\cline { 2 - 6 } & $Q_{W}$ & 0.7527 & 0.7549 & 0.7557 & 0.7609 \\
\cline { 2 - 6 } & $Q_{E}$ & 0.5716 & 0.5742 & 0.5781 & 0.5857 \\
\cline { 2 - 6 } & $Q_{A B / F}$ & 0.5825 & 0.5856 & 0.5888 & 0.5976 \\
\cline { 2 - 6 } & $\mathrm{MI}$ & 4.6626 & 4.6709 & 4.6722 & 4.6774 \\
\cline { 2 - 6 } & $\mathrm{CT}(\mathrm{sec})$ & 2.3667 & 3.1287 & 3.2484 & 2.0140 \\
\hline
\end{tabular}

Table 2. The objective evaluation results for MR-T1 and MR-T2 image dataset

\begin{tabular}{|c|c|c|c|c|c|}
\hline Image Set & Metric & NSCT-1 [1] & NSCT-2 [4] & NSCT-3 [6] & Proposed \\
\hline \multirow{4}{*}{ Image set 1 } & $Q_{0}$ & 0.6383 & 0.6389 & 0.6395 & 0.6846 \\
\cline { 2 - 6 } & $Q_{W}$ & 0.8248 & 0.8265 & 0.8276 & 0.8311 \\
\cline { 2 - 6 } & $Q_{E}$ & 0.6239 & 0.6257 & 0.6268 & 0.6284 \\
\cline { 2 - 6 } & $Q_{A B / F}$ & 0.6450 & 0.6454 & 0.6461 & 0.6490 \\
\cline { 2 - 6 } & $\mathrm{MI}$ & 5.4616 & 5.4408 & 5.4328 & 5.4741 \\
\cline { 2 - 6 } & $\mathrm{CT}(\mathrm{sec})$ & 3.3437 & 4.1344 & 4.2089 & 3.0052 \\
\hline \multirow{4}{*}{ Image set 2 } & $Q_{0}$ & 0.6311 & 0.6317 & 0.6323 & 0.6774 \\
\cline { 2 - 6 } & $Q_{W}$ & 0.8176 & 0.8193 & 0.8204 & 0.8239 \\
\cline { 2 - 6 } & $Q_{E}$ & 0.6167 & 0.6185 & 0.6196 & 0.6212 \\
\cline { 2 - 6 } & $Q_{A B / F}$ & 0.6378 & 0.6382 & 0.6389 & 0.6418 \\
\cline { 2 - 6 } & $\mathrm{MI}$ & 5.4544 & 5.4336 & 5.4256 & 5.4669 \\
\cline { 2 - 6 } & $\mathrm{CT}(\mathrm{sec})$ & 3.3365 & 4.1275 & 4.2014 & 2.9968 \\
\hline
\end{tabular}

Along with these metrics, the performance of proposed approach is also evaluated through the computational time (CT). i.e., the amount of time taken for the complete method evaluation. These performance metrics are evaluated for all the above test image sets and are formulated in Tables 1 and 2.

Fig. 2 shows the resuts obtained on the accomplishment of proposed approach over CT amd MRI image dataset. Fig.2 (a1)-(a2) are the source images A and Fig.2 (b1)-(b2) are the source images B and Fig.2 (c1)-(c2), (d1)-(d2), (e1)-(e2) represents the fused images obtained through conventional NSCT approaches. Fig.2 (f1)-(f2) show sthe obtained fused images through the proposed approach. From these two figures, it can be observed that the obtained fused image has more clear edges compared to the conventional approaches. The respective numerical results are tabulated in table.1. From the table.1, it can noticed that the proposed approach having more efficiency with respect to all the metrics. Though the increment observed in all metrics is less, the computational time taken for the process of evaluation is observed to be very less.
Since the accomplishment of a new sub band adaptive filtering technique in the proposed approach, the fusion process considers only few number of sub band images in which the improtant infomration is present, and recues most of the redundant information in the high frequency sub band images. The similar results for MR-T1 and MR-T2 image dataset is shown in Fig. 3 and its respective numerical metrics are represneted in table.2. From Fig. 3 (f), it can be observed that the proposed methods preserve both better local edge and texture information, which isthe vital information for diagnosis. The source image Fig.3 (b4) is the sub-acute premature hematoma of head, has clear edges and contours and almost all the features are replicated in the fused image obtained through proposed approach, in Fig.3 (f4).

Further the simulation is carried out by adding the noise to the both source images and then they are subjected to fusion thoruhgh the proposed and conventional approaches and the obtained fused images are shown in Figs. 4 and 5. Fig. 4 shows the 
Table.3. the objective evaluation results for in the case of noise acquisition

\begin{tabular}{|c|c|c|c|c|c|}
\hline Image Set & Metric & NSCT-1 [1] & NSCT-2 [4] & NSCT-3 [6] & Proposed \\
\hline \multirow{4}{*}{ CT and MRI } & $Q_{0}$ & 0.5767 & 0.5779 & 0.5808 & 0.6278 \\
\cline { 2 - 6 } & $Q_{W}$ & 0.7321 & 0.7343 & 0.7351 & 0.7403 \\
\cline { 2 - 6 } & $Q_{E}$ & 0.5510 & 0.5536 & 0.5575 & 0.5651 \\
\cline { 2 - 6 } & $Q_{A B / F}$ & 0.5619 & 0.5650 & 0.5682 & 0.5770 \\
\cline { 2 - 6 } & $\mathrm{MI}$ & 4.6420 & 4.6503 & 4.6516 & 4.6599 \\
\hline \multirow{4}{*}{\begin{tabular}{c} 
MR-T1 and \\
\cline { 2 - 6 }
\end{tabular}} & $Q_{0}(\mathrm{sec})$ & 2.5463 & 3.2244 & 3.3471 & 2.1127 \\
\cline { 2 - 6 } & $Q_{W}$ & 0.6279 & 0.6285 & 0.6291 & 0.6742 \\
\cline { 2 - 6 } & $Q_{E}$ & 0.8144 & 0.8161 & 0.8172 & 0.8207 \\
\cline { 2 - 6 } & $Q_{A B / F}$ & 0.6135 & 0.6153 & 0.6164 & 0.6189 \\
\cline { 2 - 6 } & $\mathrm{MI}$ & 0.6346 & 0.6352 & 0.6358 & 0.6386 \\
\hline
\end{tabular}

fused images for CT and MRI image data set and the figure.5 shows the fusd results of MR-T1 and MRT2 image dataset, clearly implies that the fused image by proposed method has better quality and contrast than other methods.

\section{Conclusion}

Medical image fusion plays important role in clinical applications. But the real challenge is to obtain a visually enhanced image through fusion process. In this paper, a new medical image fusion approach is proposed based on Non-Subsampled Contourlet Transform and Adaptive sub band filtering. Here the Non-Subsampled Contourlet Transform decomposes the image into sub band images and the ASF selects the appropriate sub band images for fusion by which the computational time reduces. As the number of levels of decomposition increases, the redundant information in the high frequency subband images also increases. If all the decomposed bands are considered for fusion, the unnecessary complexity increases. Thus the proposed ASF tends to reduce the redundant information and also helps in the removal of external noises of the source images. Further the low frequency sub band images are fused through phase congruency rule and the high frequency subband images are fused through Log-Gabor energy rule.

The experiments results indicate that the proposed fusion approach can achieve better results than the conventional fusion methods in both subjective and objective aspects.

On an average the $Q_{0}$ for proposed approach is observed to be 0.6477 , where it is of 0.5965 for NSCT [1], 0.5978 for NSCT-2 [4] and 0.6004 for NSCT-3 [6].

On an average the $Q_{W}$ for proposed approach is observed to be 0.7602 , where it is of 0.7520 for NSCT [1], 0.7542 for NSCT-2 [4] and 0.7550 for NSCT-3 [6].
On an average the $Q_{E}$ for proposed approach is observed to be 0.5850 , where it is of 0.5709 for NSCT [1], 0.5735 for NSCT-2 [4] and 0.5774 for NSCT-3 [6].

On an average the $Q_{A B / F}$ for proposed approach is observed to be 0.5969 , where it is of 0.5818 for NSCT [1], 0.5849 for NSCT-2 [4] and 0.5881 for NSCT-3 [6].

On an average the MI for proposed approach is observed to be 4.6766 , where it is of 4.6619 for NSCT [1], 4.6702 for NSCT-2 [4] and 4.6715 for NSCT-3 [6].

Finally, on an average the Computation Time for proposed approach is observed to be $2.0083 \mathrm{sec}$, where it is of $2.3360 \mathrm{sec}$ for NSCT [1], $3.128 \mathrm{sec}$ for NSCT-2 [4] and $3.2471 \mathrm{sec}$ for NSCT-3 [6].

From the above analysis, it can be observed that the proposed attained a greatest improvement in the computation time. The computation time of the proposed approach is observed to be very less and it is mainly due to the reduction in the number of processing bands, whereas the conventional approaches didn't focused on the redundant information. Hence the proposed approach is robust for multimodal medical image fusion.

In the future, the proposed approach can be extended to accomplish through a new spectral feature based band selection approach by which there is a possibility of more reduction in the computational time followed by Quality enhancement in the fused image.

\section{References}

[1] P. S. Gomathi and B. Kalaavathi, "Multimodal medical image fusion in non-subsampled contourlet transform domain", Circuits \& Systems, Vol. 7, No. 8, pp.1598-1610, 2016.

[2] M. D. C. Vald'es Hern’andez, K.J. Ferguson, F.M. Chappell, and J.M. Wardlaw, "New multispectral MRI data fusion technique for 
white matter lesion segmentation: method and comparison with thresholding in FLAIR images", European Radiology, Vol.20, No. 7, pp. 1684-1691, 2010.

[3] Z. Liu, H. Yin, Y. Chai, and S.X. Yang, "A novel approach for multimodal medical image fusion”, Expert Systems with Applications, Vol. 41, No. 16, pp. 7425-7435, 2014.

[4] Y. Yang, S.Tong, S. Huang, and P.Lin, "LogGabor energy based multimodal medical image fusion in NSCT domain", Computational and Mathematical Methods in Medicine, Vol. 2014, pp.1-12, 2014.

[5] V.D. Calhoun and T. Adali, "Feature-based fusion of medical imaging data", IEEE Transactions on Information Technology in Biomedicine, Vol. 13, No. 5, pp. 711-720, 2009.

[6] P. Ganasala and V. Kumar, "CT and MR image fusion scheme in non-subsampled contourlet transform domain", Journal of Digital Imaging, Vol. 27, No. 3, pp. 407-418, 2014.

[7] R. Shen, I. Cheng, and A. Basu, "Cross-scale coefficient selection for volumetric medical image fusion", IEEE Transactions on Biomedical Engineering, Vol. 60, No. 4, pp. 1069-1079, 2013.

[8] Z. Wang and Y. Ma, "Medical image fusion using m-PCNN", Information Fusion, Vol. 9, No. 2, pp. 176-185, 2008.

[9] N. Mitianoudis and T. Stathaki, "Optimal contrast correction for ICA-based fusion of multimodal images", IEEE Sensors J., Vol. 8, No. 12, pp. 2016-2026, 2008.

[10] V. Aslantas and R. Kurban, "Fusion of multifocus images using differential evolution algorithm", Expert Syst. Appl., Vol. 37, No. 12, pp. 8861-8870, 2010.

[11] I. De and B. Chanda, "Multi-focus image fusion using a morphologybased focus measure in a quad-tree structure", Inf. Fusion, Vol. 14, Vo. 2, pp. 136-146, 2013.

[12] S. Li, J. T. Kwok, and Y. Wang, "Multi-focus image fusion using artificial neural networks", Pattern Recognit. Lett., Vol. 23, No. 8, pp. 985-997, 2002.

[13] J. Tian, L. Chen, L. Ma, and W. Yu, "Multifocus image fusion using a bilateral gradientbased sharpness criterion", Opt. Commun., Vol. 284, No. 1, pp. 80-87, 2011.

[14] S. Li, X. Kang, J. Hu, and B. Yang, "Image matting for fusion of multi-focus images in dynamic scenes", Inf. Fusion, Vol. 14, No. 2, pp. 147-162, 2013.

[15] Y. Liu, J. Jin, Q. Wang, Y. Shen, and X. Dong, "Region level based multi-focus image fusion using quaternion wavelet and normalized cut", Signal Process., Vol. 97, pp. 9-30, 2014.

[16] G. Bhatnagar, Q. M. J. Wu, and Z. Liu, "Human visual system inspired multi-modal medical image fusion framework", Expert Systems with Applications, Vol. 40, No. 5, pp. 1708-1720, 2013.

[17] L. Yang, B. L. Guo, and W. Ni, "Multimodality medical image fusion based on multiscale geometric analysis of contourlet transform", Neuro computing, Vol. 72, No. 1-3, pp. 203211, 2008.

[18] K. Kannan, S. A. Perumal, and K. Arulmozhi, "Area level fusion of multi-focused images using multi-stationary wavelet packet transform", Int. J. Comput. Appl., Vol. 2, No. 1, pp. 88-95, 2010.

[19] S. Li, J. T. Kwok, and Y. Wang, "Using the discrete wavelet frame transform to merge Landsat TM and SPOT panchromatic images", Inf. Fusion, Vol. 3, No. 1, pp. 17-23, 2002.

[20] H. Huang, X. Feng, and J. Jiang, "Medical Image Fusion Algorithm Based on Nonlinear Approximation of Contourlet Transform and Regional Features", Journal of Electrical and Computer Engineering, Vol. 2017, pp.1-9, 2017,

[21] B. Yang and S. Li, "Multi-focus image fusion and restoration with sparse representation", IEEE Trans. Instrum. Meas., Vol. 59, No. 4, pp. 884-892, 2010.

[22] V. Bhateja, A. Srivastava, A. Moin, and A. Lay-Ekuakille, "NSCT based multispectral medical image fusion model", In: Proc. of the IEEE International Symposium on Medical Measurements and Applications, Benevento, Italy, pp. 1-5, 2016.

[23] G. Bhatnagar, Q. M. J. Wu, and Z. Liu, "A new contrast based multimodal medical image fusion framework", Neurocomputing, Vol. 157, No.1, pp. 143-152, 2015.

[24] Z. Y. Jin and Y. J.Wang, "Multi-modality medical image fusion method based on nonsubsampled contourlet transform", Chinese Journal of Medical Physics, Vol. 33, No. 5, pp. 445-450, 2016.

[25] T. Z. Xiang, R. R. Gao, and L. Yan, "MultiScale fusion of infrared and visible images based on PCNN", Journal of Geomatics Science and Technology, Vol. 3, No.2, pp. 273-278, 2016.

[26] M. N. Do and M. Vetterli, "The contourlet transform: an efficient directional multiresolution image representation", IEEE 
Transactions on Image Processing, Vol. 14, No. 12, pp. 2091-2106, 2005.

[27] G. Yang, M. Li, L. Chen, and J. Yu, "The Nonsubsampled Contourlet Transform Based Statistical Medical Image Fusion Using Generalized Gaussian Density", Computational and Mathematical Methods in Medicine, Vol. 2015, pp.1-13, 2015.

[28] A. L. da Cunha, J. Zhou, and M. N. Do, "The non-subsampled contourlet transform: theory, design, and applications", IEEE Transactions on Image Processing, Vol. 15, No. 10, pp. 3089-3101,2006.

[29] K. Aik, W.S.G. Nanyang, S.M. Kuo, "Subband Adaptive Filtering Theory and Implementation", Wiley and Sons publishers, 2009.

[30] M.K. Song, S.E. Kim, Y.S. Choi, and W.J. Song, "A Selective Normalized Subband Adaptive Filter Exploiting an efficient subset of Sub bands", In: Proc. of European Signal Processing Conference, 2011.

[31] M.S.E. Abadi, and M.S. Shafiee, "A New Variable Step-Size Normalized Subband Adaptive Filter Algorithm Employing Dynamic Selection of Subband Filters", In: Proc. of International Conf. On Electrical Engineering (ICEE), 2013.

[32] Y. Yang, S. Tong, S. Huang, and P. Lin, "Multi-focus Image Fusion Based on NSCT and Focused Area Detection", IEEE Sensors Journal, Vol. 15, No. 5, pp.2824-2838, 2015.

[33] C. Yang, "A novel similarity based quality metric for image fusion", Inf. fusion, Vol. 9, No. 2, pp. 156-160, 2008.

[34] G. Piella and H. Heijmans, "A new quality metric for image fusion," In: Proc. of the International Conference on Image Processing (ICIP '03), Catalonia, Spain, pp. 173-176, 2003.

[35] C. S. Xydeas and V. Petrovi'c, "Objective image fusion performance measure", Electronics Letters, Vol. 36, No. 4, pp. 308309, 2000.

[36] G. Qu, D. Zhang, and P. Yan, "Information measure for performance of image fusion", Electronics Letters, Vol. 38, No. 7, pp. 313315, 2002.

[37] A.R. Sanjay, R.K. Soundrapandiyan, M. Karuppiah, and R. Ganapathy, "CT and MRI Image Fusion Based on Discrete Wavelet Transform and Type-2 Fuzzy Logic", International Journal of Intelligent Engineering and Systems, Vol. 10, No. 3, pp. 355-362, 2017. 